\title{
The Effects of Couple Stresses in the Squeeze Film Characteristics Between a Cylinder and a Plane Surface
}

\author{
Jaw-Ren Lin \\ Professor, Department of Mechanical Engineering, Nanya Institute of Technology, P.O. Box 324-22-59, Chung-Li, \\ Taiwan, R.O.C., jrlin@nanya.edu.tw \\ Won-Hsion Liao \\ Instructor, Department of Mechanical Engineering, Nanya Institute of Technology, P.O. Box 324-22-59, Chung-Li, \\ Taiwan, R.O.C. \\ Chi-Ren Hung \\ Instructor, Department of Mechanical Engineering, Nanya Institute of Technology, P.O. Box 324-22-59, Chung-Li, \\ Taiwan, R.O.C.
}

Follow this and additional works at: https://jmstt.ntou.edu.tw/journal

Part of the Mechanical Engineering Commons

\section{Recommended Citation}

Lin, Jaw-Ren; Liao, Won-Hsion; and Hung, Chi-Ren (2004) "The Effects of Couple Stresses in the Squeeze Film Characteristics Between a Cylinder and a Plane Surface," Journal of Marine Science and Technology. Vol. 12: Iss. 2, Article 7.

DOI: $10.51400 / 2709-6998.2228$

Available at: https://jmstt.ntou.edu.tw/journal/vol12/iss2/7

This Research Article is brought to you for free and open access by Journal of Marine Science and Technology. It has been accepted for inclusion in Journal of Marine Science and Technology by an authorized editor of Journal of Marine Science and Technology. 


\title{
Short Paper
}

\section{THE EFFECTS OF COUPLE STRESSES IN THE SQUEEZE FILM CHARACTERISTICS BETWEEN A CYLINDER AND A PLANE SURFACE}

\author{
Jaw-Ren Lin*, Won-Hsion Liao**, and Chi-Ren Hung**
}

Key words: micro-continuum theory, squeeze films, cylinder and plane surface, couple stress fluids.

\section{ABSTRACT}

On the basis of the Stokes micro-continuum theory, the effects of couple stresses resulting from the lubricant blended with various additives upon the squeeze film behavior between a long cylinder and a plane surface are analyzed. Compared to the conventional Newtonianlubricant case, the couple stress effects characterized by the couple stress parameter signify an improvement in the squeeze film characteristics. Increasing values of the couple stress parameter increases the hydrodynamic load-carrying capacity and provides a longer time to prevent cylinder-plane surface contact. The approaching time of the cylinder in reducing the film thickness $h_{m}^{*}=1$ to $h_{m}^{*}$ $=0.01$ for the couple stress fluid lubricant is about 27.67 , which is longer than the approaching time 22.62 for the case of a Newtonian lubricant.

\section{INTRODUCTION}

The squeezing-film technology is widely observed in applications of engineering practices such as human joints, dampers, matching gears, and machine-tool systems. Traditionally, the study of squeeze film systems concentrates upon the use of a Newtonian viscous fluid. Many types of mechanisms are analyzed by Hamrock [2], and Pinkus and Sternlicht [10]. Owing to the developing of modern machine apparatus, the increasing application of fluids containing microstructures such as suspensions or long-chained polymers has been emphasized. To describe the flow characteristics of these kinds of non-Newtonian fluids, a micro-continuum theory of couple stress fluids have been pro-

Paper Submitted 01/02/04, Accepted 04/29/04. Author for Correspondence: Jaw-Ren Lin. E-mail: jrlin@nanya.edu.tw.

*Professor, Department of Mechanical Engineering, Nanya Institute of Technology, P.O. Box 324-22-59, Chung-Li, Taiwan, R.O.C.

**Instructor, Department of Mechanical Engineering, Nanya Institute of Technology, P.O. Box 324-22-59, Chung-Li, Taiwan, R.O.C. posed by Stokes [12]. By the application of this couple stress fluid model, various researches regarding the hydrodynamic thin-film problems have been carried out. Representative works are presented in the studies of journal bearings by Lin $[4,5,8]$, the slider bearings by Lin et al [9], the squeeze film plates of various shapes by Ramanaiah [11], the squeeze-film configuration with reference to synovial joints by Bujurke and Jayaraman [1] and Lin [3], the squeeze-film partial journal bearings by Lin [6], and the squeeze film behavior between a sphere and a flat plate [7]. It is known that the squeeze film behavior between a cylinder and a plane surface lubricated with a Newtonian lubricant has been analyzed [2]. However, we have no idea how the couple stress effects affect the cylinder-plane system. By this way, a further study is motivated.

\section{ANALYSIS}

Based upon the Stokes couple stress fluid model, the effects of couple stresses on the squeeze film characteristics between a cylinder and a plane surface is analyzed. Comparing with the classical Newtonianlubricant case, the squeeze film characteristics are presented for various couple stress parameter.

The squeeze film geometry is considered in Figure 1. A long rigid cylinder of radius $R$ is approaching an infinite plane surface plate with a velocity $V=-\partial h / \partial t$. The lubricant is taken to be a Stokes couple stress fluid. Under the assumption that the fluid film is thin [2], the fluid inertia is small, and the body forces and body couples are absent, the continuity equation and the motion equations reduce to the follows.

$$
\begin{aligned}
& \frac{\partial u}{\partial x}+\frac{\partial w}{\partial z}=0 \\
& \frac{\partial p}{\partial x}=\mu \frac{\partial^{2} u}{\partial z^{2}}-\eta \frac{\partial^{4} u}{\partial z^{4}} \\
& \frac{\partial p}{\partial z}=0
\end{aligned}
$$


where $\eta$ is a new material constant responsible for the couple stress fluid property with the dimension of momentum. Its value could be determined by some experiments as discussed by Stokes [12]. The boundary conditions for $u(x, z)$ are:

$$
\left.u\right|_{z=0}=\left.\frac{\partial^{2} u}{\partial z^{2}}\right|_{z=0}=0,\left.\quad u\right|_{z=h}=\left.\frac{\partial^{2} u}{\partial z^{2}}\right|_{z=h}=0
$$

The film thickness $h$, because in the film region $x$ $<R$, is approximated by [2]

$$
h=h_{m}+\frac{x^{2}}{2 R}
$$

where $h_{m}$ denotes the minimum film thickness. Solving equation (2) with the above boundary conditions one can obtain the expression of $u$.

$$
u(x, z)=\frac{1}{2 \mu} \frac{d p}{d x}\left\{z^{2}-h z+2 l^{2}\left\{1-\frac{\cos h[(2 z-h) /(2 l)]}{\cos h[h /(2 l)]}\right\}\right\}
$$

where

$$
l=(\eta / \mu)^{1 / 2}
$$

Since the dimension of $\eta$ is of momentum, the dimension of $l$ is therefore of length. This length of $l$ could be considered as the characteristic material length or the molecular length of the polar suspensions in a non-polar fluid. Now integrate the continuity equation (1) with respect to $z$ with the boundary conditions of $w(x, z)$ :

$$
\left.w\right|_{z=0} \text { and }\left.w\right|_{z=h}=\frac{\partial h}{\partial t}
$$

The modified Reynolds-type equation governing the squeeze film pressure for the system is obtained [6].

$$
\frac{\partial}{\partial x}\left\{\left[h^{3}-12 l^{2} h+24 l^{3} \tan h\left(\frac{h}{21}\right)\right] \frac{d p}{d x}\right\}=12 \mu \frac{\partial h}{\partial t}
$$

Introduce the dimensionless variables and parameters:

$$
\begin{aligned}
& x^{*}=\frac{x}{R}, p^{*}=\frac{p h_{m 0}^{2}}{\mu R(-\partial h / \partial t)}, h_{m}^{*}=\frac{h_{m}}{h_{m 0}}, \\
& l^{*}=\frac{l}{h_{m 0}}, \beta=\frac{h_{m 0}}{R}
\end{aligned}
$$

where $h_{m 0}$ represents the initial minimum film thickness. The dimensionless modified Reynolds-type equation is then expressed as the following.

$$
\frac{\partial}{\partial x^{*}}\left[f^{*}\left(h^{*}, l^{*}\right) \frac{d p^{*}}{d x^{*}}\right]=-\frac{12}{\beta}
$$

where the functions $h^{*}$ and $f^{*}$ are defined by:

$$
\begin{aligned}
& h^{*}=\frac{h}{h_{m 0}}=h_{m}^{*}+\frac{x^{* 2}}{2 \beta} \\
& f^{*}\left(h^{*}, l^{*}\right)=h^{* 3}-12 l^{*^{2}} h^{*}+24 l^{*} \tan h\left(\frac{h^{*}}{2 l^{*}}\right)
\end{aligned}
$$

The boundary conditions for the film pressure are: $p^{*}=0$ at $x^{*}=1$ and $d p^{*} / d x^{*}=0$ at $x^{*}=0$. Integrating the Reynolds equation with respect to $x^{*}$ with the above conditions, the squeeze film pressure is obtained.

$$
p^{*}=\frac{12}{\beta} \int_{x^{*}}^{x^{*}=1} \frac{x^{*}}{f^{*}\left(h^{*}, x^{*}\right)} d x^{*}
$$

\section{SQUEEZE FILM CHARACTERISTICS}

By integrating the film pressure acting upon the cylinder we can compute load-carrying capacity.

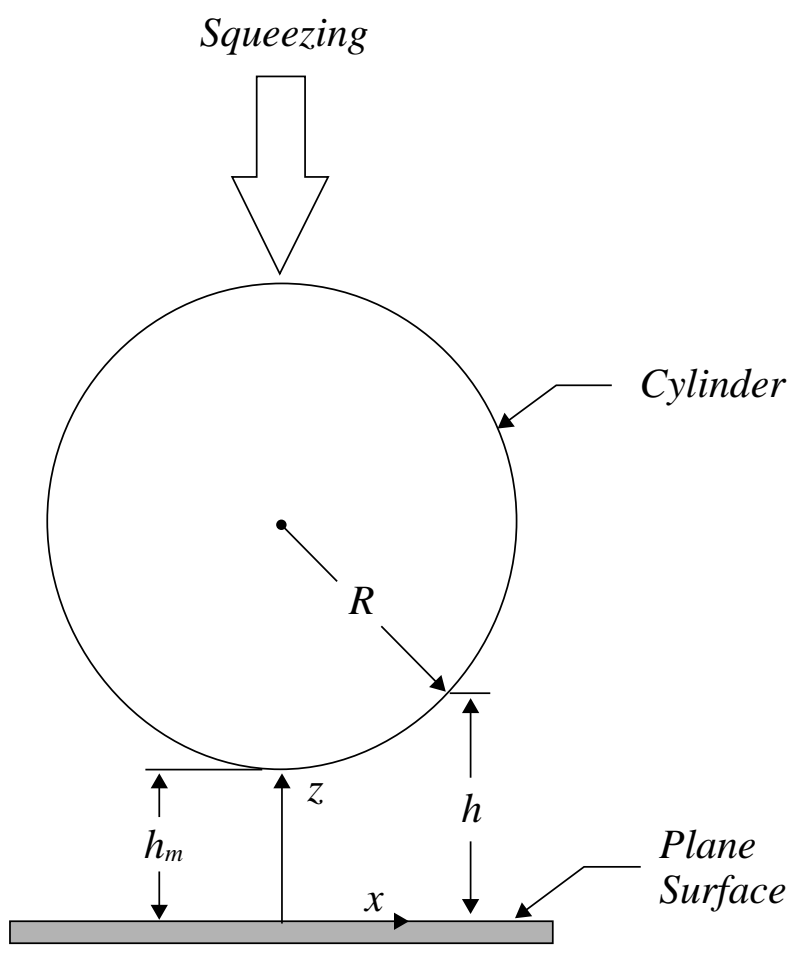

Fig. 1. Squeez film geometry between a cylinder and a plane surface. 


$$
W=\int_{x=-R}^{R} p B d x
$$

where $B$ denotes the length of the cylinder. Expressing in a dimensionless form results in

$$
W^{*}=\frac{W h_{m 0}^{2}}{\mu R^{2} B(-\partial h / \partial t)}=\frac{12}{\beta} \int_{x^{*}=-1}^{1} \frac{x^{* 2}}{f^{*}\left(h^{*}, l^{*}\right)} d x^{*}
$$

The time of approach can be obtained by integrating the above equation with the initial condition: $h_{m}(t=$ 0) $h_{m_{0}}$. Performing the integration and expressing in a dimensionless form and one can obtain the dimensionless time of approach.

$$
t^{*}=\frac{W h_{m_{0}}^{2}}{\mu R^{3} B} t=12 \int_{h_{m}^{*}}^{h_{m}^{*}=1}\left[\int_{x^{*}=-1}^{1} \frac{x^{* 2}}{f^{*}\left(h^{*}, l^{*}\right)} d x^{*}\right] d h_{m}^{*}
$$

Although the values of the film pressure, the loadcarrying capacity and the time of approach in equations (14), (16) and (17) cannot be calculated by direct integration, they could be numerically evaluated by the method of Gaussian quadrature.

\section{RESULTS AND DISCUSSION}

From the Stokes theory, the new material constant $\eta$ in equation (2) is responsible for the property of couple stresses. Since the dimension of $l$ in equation (7) is of length, it may be identified as the characteristic length of additives in a Newtonian lubricant. Consequently, the effects of couple stresses are characterized by the couple stress parameter $l^{*}$ defined in equation (10). As the value of $l^{*}$ approaches zero, the dimensionless modified Reynolds-type equation reduces to the Newtonian-lubricant case $[2,10]$. To obtain the squeeze film characteristics, the results are presented for the parameters with $\beta=0.05$ and $l^{*}=0 \sim 0.5$. The dimensionless film pressure $p^{*}$ as a function of $x^{*}$ for different values of $l^{*}$ is shown in Figure 2. It is seen that the couple stress effects result in a higher film pressure, especially in the vicinity of the position of minimum film height. Figure 3 presents the dimensionless maximum film pressure $p_{\max }^{*}$ as a function of $h_{m}^{*}$ for different values of $l^{*}$. Compared with the Newtonian-lubricant case, the effects of couple stresses signify an increase in the value of $p_{\max }^{*}$. In addition, the lower the minimum squeeze film height, the more the couple stresses affect the maximum film pressure.

Figure 4 presents the dimensionless load-carrying capacity $W^{*}$ as a function of dimensionless minimum film height $h_{m}^{*}$ for different values of couple stress parameter $l^{*}$. Since the couple stress effects results in a higher film pressure, the integrated load-carrying is similarly affected. Compared with the Newtonianlubricant case, the effects of couple stresses are observed to increase the load-carrying capacity. Figure 5 describes the dimensionless load-carrying capacity $W^{*}$

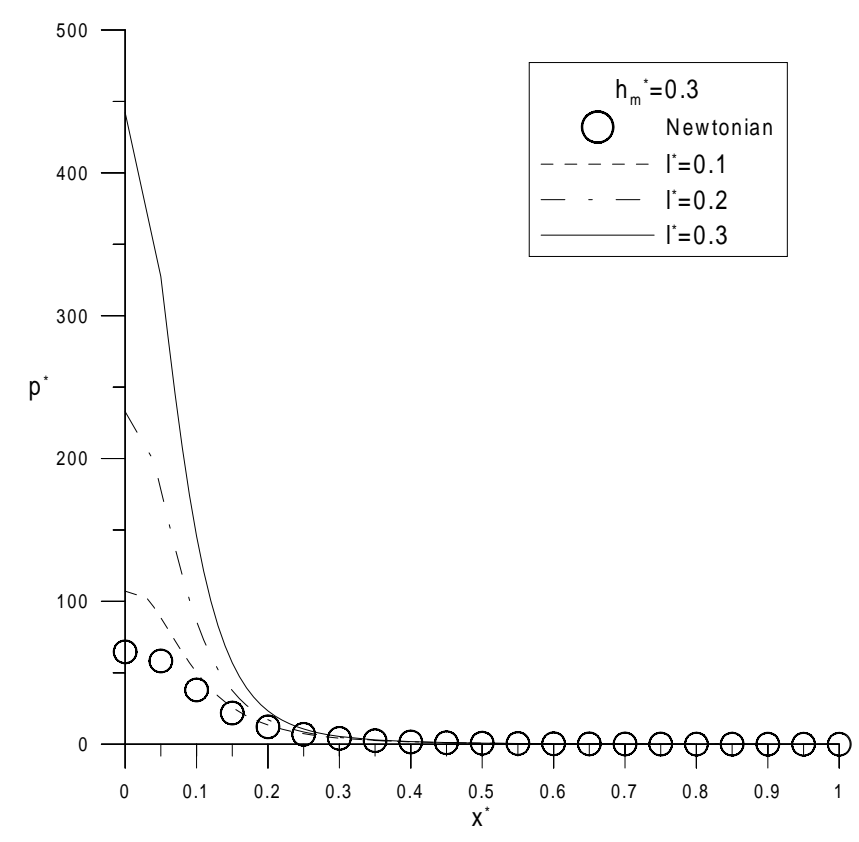

Fig. 2. Dimensionless film pressure $p^{*}$ as a function of $x^{*}$ for different $l^{*}$.

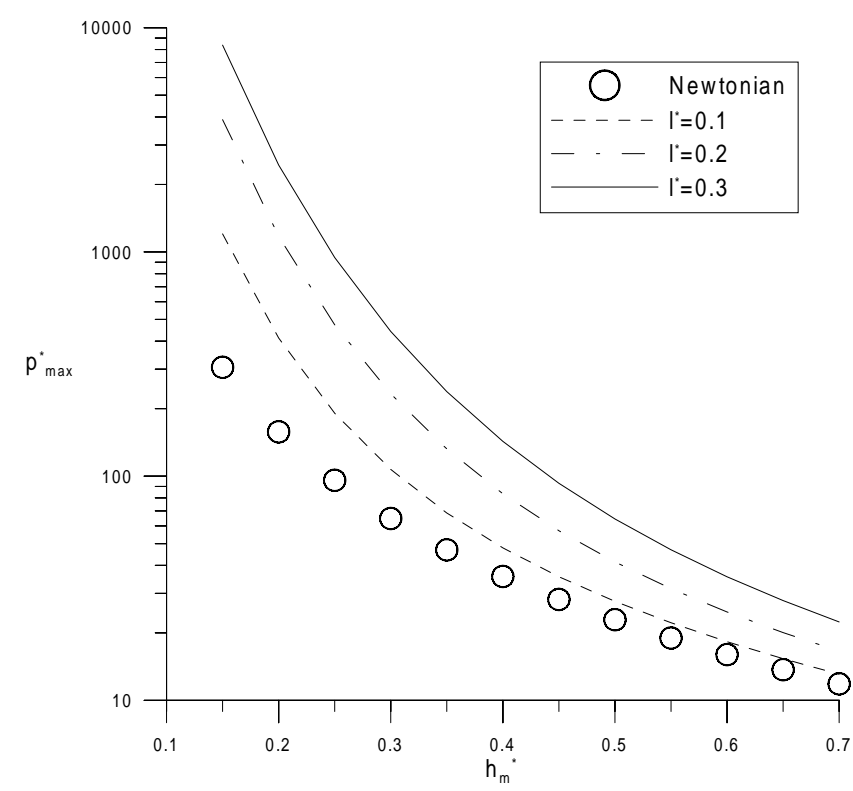

Fig. 3. Dimensionless maximum pressure $p_{\max }^{*}$ as a function of $h_{m}^{*}$ for different $l^{*}$. 
as a function of couple stress parameter $l^{*}$ at various dimensionless minimum film heights $h_{m}^{*}$. As shown, the effects of couple stresses produce an increase in the load-carrying capacity. Moreover, a larger increment of the load is obtained with increasing value of $l^{*}$ or decreasing value of $h_{m}^{*}$. Figure 6 shows the dimension-

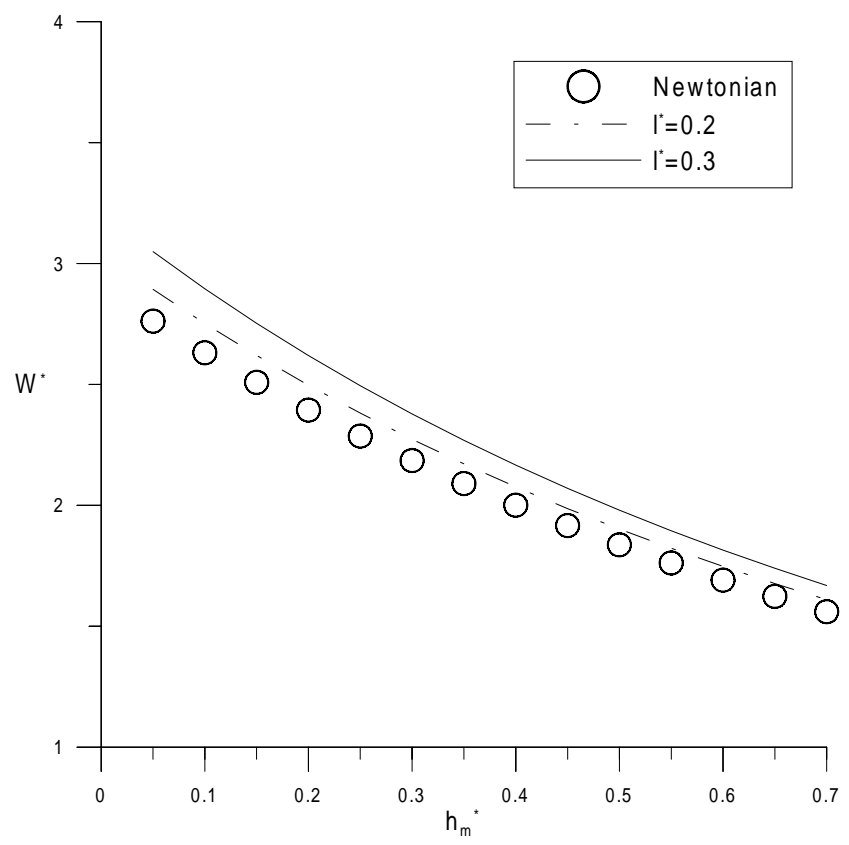

Fig. 4. Dimensionless load-carrying capacity $W^{*}$ versus $h_{m}^{*}$ for different $l^{*}$.



Fig. 5. Dimensionless load-carrying Capacity $W^{*}$ versus $l^{*}$ for different $h_{m}^{*}$. less minimum film height $h_{m}^{*}$ as a function of dimensionless time of approach $t^{*}$ for different values of couple stress parameter $l^{*}$. It is seen that the presence of couple stresses provides an increase in the time of approach. These phenomena can be realized that since the couple stress effects yield a higher load-carrying capacity, a higher $h_{m}^{*}$ would be attained for the same time to be taken as compared to the Newtonian-lubricant case. In other words, the time of approach for the cylinder-plane surface system is lengthened by the use of an incompressible couple stress fluid.

\section{CONCLUSIONS}

On the basis of Stokes micro-continuum theory, the effects of couple stresses on the squeeze film motion between a cylinder and a plane surface are presented. To account for the couple stress effects resulting from a Newtonian lubricant blended with various additives, the modified Reynolds-type equation is derived using the Stokes motion equations. According to the results discussed, conclusions can be drawn as follows. The presence of couple stresses characterized by the couple stress parameter has a significant effect upon the squeezefilm system considered. Comparing with the classical Newtonian-lubricant case, increasing the value of the couple stress parameter provides an increase in the film pressure and the load-carrying capacity, and thereby provides a longer time to prevent the cylinder-plane surface contact. The approaching time of the cylinder in

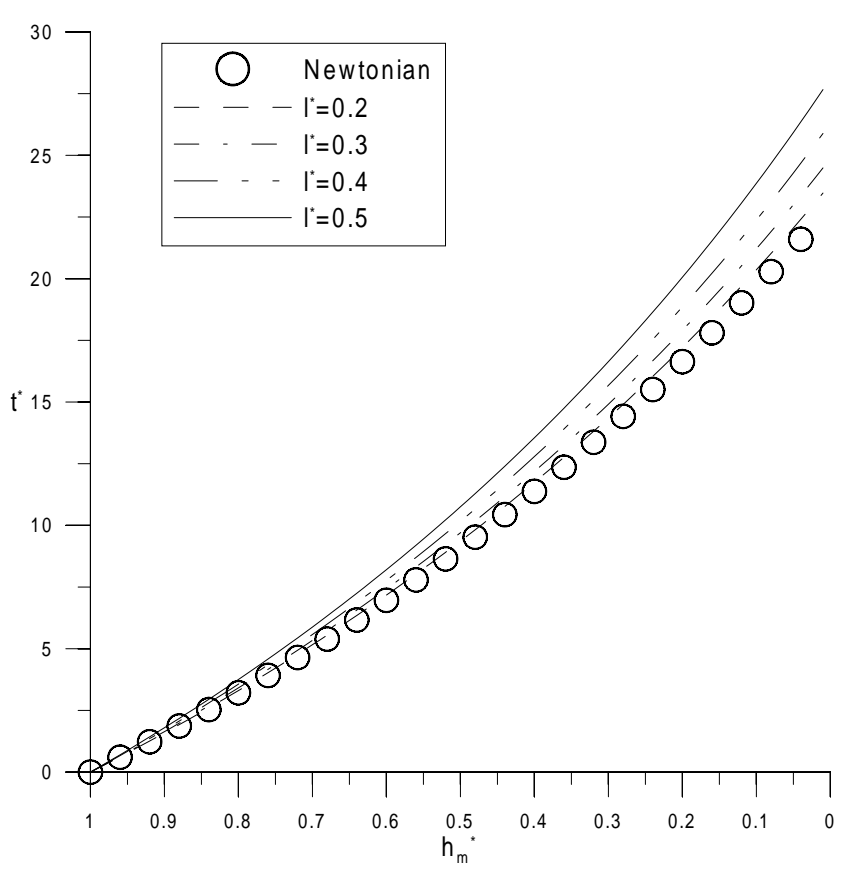

Fig. 6. Dimensionless minimum film height $h_{m}^{*}$ versus $t^{*}$ for different $l^{*}$. 
reducing the film thickness $h_{m}^{*}=1$ to $h_{m}^{*}=0.01$ for the couple stress fluid lubricant $l^{*}=0.5$ is about 27.67 , which is longer than the approaching time 22.62 for the case of a Newtonian lubricant.

\section{REFERENCES}

1. Bujurke, N.M. and Jayaraman, G., "The Influence of Couple Stresses in Squeeze Films," Int. J. Mech. Sci., Vol. 24, pp. 369-76 (1982).

2. Hamrock, B.J., Fundamentals of Fluid Film Lubrication, McGraw-Hill, Inc., New York (1994).

3. Lin, J.R., "Couple-stress Effects on the Squeeze Film Characteristics of Hemispherical Bearings with Reference to Synovial Joints," Int. J. Appl. Mech. Eng., Vol. 1, pp. 317-332 (1996).

4. Lin, J.R., "Effects of Couple Stresses on the Lubrication of Finite Journal Bearing," Wear, Vol. 206, pp.171-178 (1997).

5. Lin, J.R., "Static Characteristics of Rotor Bearing System Lubricated with Couple Stress Fluids," Computers Struct., Vol. 62, No. 1, pp. 175-184 (1997).
6. Lin, J.R., "Squeeze Film Characteristics of Long Partial Journal Bearings Lubricated with Couple Stress Fluids," Tribol. Int., Vol. 30, pp. 53-58 (1997).

7. Lin, J.R., "Squeeze Film Characteristics Between a Sphere and a Flat Plate: Couple Stress Fluid Model," Computers Struct., Vol. 75, pp. 73-80 (2000).

8. Lin, J.R., "Linear Stability Analysis of Rotor Bearing System: Couple Stress Fluid Model," Computers Struct., Vol. 79, pp. 801-809 (2001).

9. Lin, J.R., Lu, R.F., and Chang, T.B., "Derivation of Dynamic Couple-stress Reynolds Equation of Sliding-squeezing Surfaces and Numerical Solution of Plane Inclined Slider Bearings," Tribol. Int., Vol. 36, pp. 679-685 (2003).

10. Pinkus, O. and Sternlicht, B., Theory of Hydrodynamic Lubrication, McGraw-Hill, Inc., New York (1961).

11. Ramanaiah, G., "Squeeze Films Between Finite Plates Lubricated by Fluids with Couple Stress," Wear, Vol. 54, pp. 315-320 (1979).

12. Stokes, V.K., "Couple Stresses in Fluids," Phys. Fluids, Vol. 9, pp. 1709-1715 (1966). 